\title{
EL NUEVO RÉGIMEN DE LA REUTILIZACIÓN DE LAS AGUAS DEPURADAS: EN ESPECIAL, LAS NOVEDADES EN EL NUEVO PLAN HIDROLÓGICO DE LA DEMARCACIÓN DEL SEGURA
}

\author{
SONIA M. HERNÁNDEZ LÓPEZ \\ Técnico facultativo superior. Confederación Hidrográfica del Segura \\ sonia.hernandez@chsegura.es \\ SANTIAGo M. Álvarez CARREÑo \\ Profesor titular de Derecho Administrativo. Universidad de Murcia \\ santialv@um.es
}

Recibido: 13 de octubre de 2014 / Aceptado: 20 de noviembre de 2014

RESUMEN: La reutilización de las aguas depuradas constituye una práctica adecuada para incrementar la disponibilidad del recurso. Sin embargo, las implicaciones sanitarias que pudiera conllevar hacen necesario la instauración de un marco jurídico que otorgue seguridad a los diferentes sectores implicados. La incorporación de las aguas regeneradas a la gestión integral del dominio público hidráulico implica, a su vez, su consideración en los nuevos planes hidrológicos recientemente aprobados. En la Demarcación Hidrográfica del Segura, donde el recurso se encuentra sometido a una intensa presión por los actuales y potenciales usuarios, la reutilización puede representar un relevante recurso adicional.

RESUM: La reutilització de les aigües depurades constitueix una pràctica adequada per incrementar la disponibilitat del recurs. Tanmateix, les implicacions sanitàries que podria comportar fan necessària la instauració d'un marc jurídic que atorgui seguretat als diferents sectors implicats. La incorporació de les aigües regenerades a la gestió integral del domini públic hidràulic implica, així mateix, llur consideració en els nous plans hidrològics aprovats recentment. En la Demarcació Hidrogràfica del Segura, on el 
recurs es troba sotmès a una intensa pressió pels usuaris actuals i potencials, la reutilització pot representar un recurs addicional.

ABSTRACT: The reuse of treated water is a good practice to increase this resource's availability. However, the potential health implications call for the establishment of a legal framework that gives security to the different sectors involved. The incorporation of treated water into the comprehensive management of public water implies, in turn, its consideration in the recently approved hydrologic management plans. In the Segura River Basin, where the resource is under intense pressure by current and potential users, reuse may represent an important additional resource.

PALABRAS CLAVE: Reutilización aguas depuradas — Planificación hidrológica Demarcación Hidrográfica del Segura.

PARAULES CLAU: Reutilització de les aigües depurades — Planificació hidrològica — Demarcació Hidrogràfica del Segura.

KEY WORDS: Reuse of treated water - Water management — Segura River Basin.

Sumario: I. Introducción: los condicionantes de la actividad de reutilización. II. Los inicios de la reutilización en el ordenamiento jurídico español: el texto refundido de la Ley de Aguas y el Real Decreto 1620/2007, de 7 de diciembre. III. La reutilización de las aguas en el nuevo Plan Hidrológico de la Demarcación del Segura. 1. Primeras apariciones de la reutilización en el nuevo Plan Hidrológico de la Demarcación del Segura. 2. Desarrollo de la reutilización de aguas depuradas en la nueva planificación del Segura. IV. Conclusiones. V. Bibliografía.

\section{INTRODUCCIÓN: LOS CONDICIONANTES DE LA ACTIVIDAD DE REUTILIZACIÓN}

Ya en 1967 el Informe del Grupo Científico de la Organización Mundial de la Salud sobre Tratamiento y Evacuación de Desechos señalaba que las aguas residuales se venían aprovechando desde hacía muchos años, especialmente en la agricultura. El transcurso del tiempo no ha hecho sino aumentar la importancia de esta reutilización tanto por su relación con la lucha contra la contaminación como por el interés que 
supone incrementar la cantidad de agua disponible para los usos agrícolas, industriales, recreativos y domésticos. En efecto, resulta indiscutible que, de todas las actuaciones que pudieran acometerse para incrementar la disponibilidad del recurso, la reutilización de las aguas depuradas es la que mayores posibilidades tiene a corto plazo ${ }^{1}$. Esto se debe a que la materia prima de las aguas regeneradas son las aguas residuales que una vez depuradas son aptas para su vertido al entorno. Esta depuración y vertido posterior controlado se realiza en España y en toda la Unión europea con elevados niveles de garantía y en plantas de tratamiento cada vez más avanzadas, impulsado en gran parte por las obligaciones derivadas de la Directiva 91/271/CEE, de 21 de mayo, sobre tratamiento de las aguas residuales urbanas. En efecto, la regeneración de las aguas depuradas no suele implicar más allá de la mera incorporación de un tratamiento adicional (desinfección) sobre las aguas ya depuradas, tratamiento que en ocasiones ya existe en las plantas de depuración. De esta forma y casi inmediatamente, se dispone de un nuevo recurso apto para ser transportado hasta las zonas de reutilización. Se hace evidente que la reutilización permite, por una parte, aumentar la oferta del recurso, disminuyendo así la presión sobre los de origen convencional (aguas superficiales y subterráneas), mientras que, por otra, reduce la contaminación del entorno al evitar el vertido de aguas residuales al medio ambiente ${ }^{2}$.

Es igualmente indiscutible que, para que una materia se desarrolle y se normalice su presencia en la ecuación global de disponibilidad del recurso, se requiere un marco jurídico estable que garantice la seguridad que exigen todos los actores administrativos, sociales y económicos que intervienen en el proceso de reutilización. Cuando se trata de la reutilización, la importancia de estos aspectos es, si cabe, aún más importante dada la trascendencia sanitaria y ambiental que conlleva un uso inadecuado de las aguas depuradas.

La importancia de las aguas regeneradas depende igualmente de su disponibilidad y facilidad de acceso por parte de los usuarios potenciales. En este sentido, es fundamental que la seguridad jurídica venga acompañada de un estudio pormenorizado

\footnotetext{
${ }^{1}$ RICO AMORÓS, A. M., "Depuración y reutilización de aguas residuales en el litoral alicantino", Papeles de Geografía, núm. 23-24, 1996, p. 248.

${ }^{2}$ HERNÁNDEZ SANCHO, F., MOLINOS-SENANTE, M. y SALA GARRIDO, R., "Estudio de la viabilidad para proyectos de reutilización de aguas residuales: valoración económica de los beneficios ambientales" 2007, p. 2.
} 
de las fortalezas y carencias en la materia y, en particular, del volumen de agua residual susceptible de ser reutilizada.

Este estudio podría incluso plantearse el límite de la reutilización, esto es, si existe un volumen disponible de agua regenerada que aún no está siendo objeto de un segundo uso. Al respecto, en España el volumen máximo está limitado por la cantidad de agua urbana depurada, la situación geográfica respecto al uso de estas instalaciones, la demanda, la aceptación del recurso y la viabilidad económica y medioambiental de este $^{3}$.

De esta forma, recientemente y dentro de los trabajos preparatorios que está realizando la Comisión Europea para el establecimiento de una norma comunitaria en materia de reutilización, se ha estimado que, conforme a los datos más recientes, el volumen total de reutilización de aguas residuales tratadas en la Unión Europea en el año 2006 era de 964 millones de $\mathrm{m}^{3}$ /año; de ellos, a España correspondían 347 millones de $\mathrm{m}^{3} /$ año, esto es, casi el $36 \%$ del total, seguida de Italia, Chipre y Malta ${ }^{4}$.

Otros estudios realizados en España en este sentido identifican las zonas donde el volumen de agua depurada que se reutiliza es mayor. Así, según la versión preliminar del Plan Nacional de Reutilización de Aguas, en el año 2006 se reutilizaron en España $28,2 \mathrm{hm}^{3}$ de agua depurada, de los cuales el $28 \%\left(104,8 \mathrm{hm}^{3}\right)$ correspondía a la demarcación hidrográfica del Segura, solo superada en porcentaje por la demarcación hidrográfica del Júcar, que reutilizó el $35 \%$ del total $\left(128,4 \mathrm{hm}^{3}\right)$.

Resulta evidente que los mayores porcentajes de reutilización se concentran en las zonas de mayor escasez, sometidas a la presión del recurso condicionado por los períodos recurrentes de sequía. Cuanto más al norte, por el contrario, el volumen de agua reutilizada se va reduciendo, desapareciendo casi por completo, según datos del Plan Nacional de Reutilización, en la cuenca cantábrica.

La Comisión Europea señala que España es el país con mayor potencial de reutilización al estimar un volumen de reutilización de 1.200 millones $\mathrm{de}^{3}$ /año de aquí a 2025 . Para su estudio se desarrolló un modelo basado en un planteamiento de balance de masas que

\footnotetext{
${ }^{3}$ MELGAREJO MORENO, J., "Efectos ambientales y económicos de la reutilización del agua en España”, CLM Economía, núm. 15, 2009, p. 250.

${ }^{4}$ COMISIÓN EUROPEA, "Documento de base de la consulta pública sobre las opciones políticas para optimizar la reutilización de agua en la UE", 2014, p. 1 (disponible en: $<$ http://ec.europa.eu/environment/water/blueprint/pdf/water_reuse>. Último acceso 27/09/2014).
} 
considera, por una parte, la cantidad de agua regenerada disponible para su reutilización y, por otra, la demanda de ese tipo de agua en los diferentes sectores de actividad.

En el caso particular de la demarcación hidrográfica del Segura, la versión preliminar del Plan Nacional de Reutilización indica que las posibilidades de desarrollo de la reutilización en la cuenca se prevén escasas por cuanto se parte de un volumen de reutilización alto y de la necesidad de asegurar los caudales ecológicos de los ríos ${ }^{5}$.

No obstante, tras la aprobación del nuevo Plan Hidrológico de la Demarcación del Segura, acompañada por otras recientes modificaciones referidas a la reutilización, el escenario parece haberse ampliado. Así, mediante Acuerdo de la Junta de Gobierno de la Confederación Hidrográfica del Segura de 15 de julio de $2013^{6}$ quedó derogado el anterior Acuerdo del día 8 de marzo de 2006 que ordenó la suspensión de la tramitación de nuevas concesiones de aguas procedentes de depuradoras de aguas residuales cuyos efluentes estuvieran próximos o pudieran ser fácilmente vertidos a las acequias o azarbes, al río Segura o a sus afluentes, así como la denegación de los que se estuvieran tramitando en la actualidad, hasta que, por cambiar el déficit continuado de la cuenca, pudieran circular los caudales ecológicos necesarios aguas arriba del azud de Rojales.

De este modo, el eje central del presente estudio gira en torno a estas nuevas aportaciones de la planificación hidrológica en este ámbito fundamental para la gestión integral del recurso, de forma que se pueda calibrar la importancia en un inmediato futuro de la reutilización de las aguas.

\section{LOS INICIOS DE LA REUTILIZACIÓN EN EL ORDENAMIENTO JURÍDICO ESPAÑOL: EL TEXTO REFUNDIDO DE LA LEY DE AGUAS Y EL REAL DECRETO 1620/2007, DE 7 DE DICIEMBRE.}

A nivel estatal, la regulación jurídica de la reutilización de las aguas aparece en el texto refundido de la Ley de Aguas aprobado mediante RD -Legislativo 1/2001, de 20 de julio (en adelante, TRLA), en su artículo 109. Según este precepto, "el Gobierno establecerá las condiciones básicas para la reutilización de las aguas, precisando la

\footnotetext{
${ }^{5}$ MINISTERIO DE MEDIO AMBIENTE, MEDIO RURAL Y MARINO, Guía para la aplicación del RD 1620/2007 por el que se establece el Régimen Jurídico de la Reutilización de las Aguas Depuradas, 2010, p. 24 (disponible en: <http://www.magrama.gob.es/es/agua>. Último acceso 29/09/2014).

${ }^{6}$ BORM, núm. 269, de 20 de noviembre de 2013.
} 
calidad exigible a las aguas depuradas según los usos previstos”. Igualmente, se precisa que el titular de la concesión o autorización de reutilización deberá sufragar los costes necesarios para adecuar las aguas a las exigencias de calidad vigentes en cada momento. Esta determinación del régimen de responsabilidades se respeta en el desarrollo posterior que lleva a cabo el Real Decreto 1620/2007, de 7 de diciembre, por el que se establece el régimen jurídico de la reutilización de las aguas depuradas. No obstante, y tal y como se analizará en un momento posterior de este estudio, la situación actual difiere en ocasiones del esquema normativo general.

Según este, los costes derivados de la adecuación de la calidad de las aguas regeneradas al uso previsto, esto es, de la infraestructura capaz de alcanzar los niveles de calidad exigidos, recae sobre el concesionario. En la realidad, y en la mayoría de las ocasiones, estos costes se imputan a entidades públicas o, incluso, a los municipios titulares de las depuradoras.

Por otro lado, el artículo 109 TRLA establece la obligación de obtener una concesión administrativa para la reutilización de aguas, salvo que sea solicitada por el titular de una autorización de vertido, en cuyo caso únicamente requerirá autorización administrativa. Esta línea general marcada por el TRLA, como no podría ser de otra manera, es respetada y desarrollada por el RD 1620/2007, que incorpora consideraciones en relación con el orden de preferencia en caso de concurrencia de proyectos. De esta forma, cuando el solicitante es el titular de la autorización de vertido, tendrá prioridad frente al primer usuario de las aguas y este, frente a terceros.

Visto lo anterior, el procedimiento administrativo para obtener el permiso de reutilización (ya sea concesión o autorización) difiere en función del tipo de solicitante. Así, cuando quien lo solicita es el primer usuario de las aguas, el artículo 8 del RD 1620/2007 establece un procedimiento administrativo cuya tramitación guarda evidentes similitudes con el establecido en el Reglamento del Dominio Público Hidráulico aprobado mediante Real Decreto 849/1986, de 11 de abril (en adelante, RDPH), para las concesiones en general, con la salvedad de que no se someterá a competencia de proyectos. Cuando quien solicita es titular de la autorización de vertido de las aguas depuradas, el artículo 9 del RD 1620/2007 establece que únicamente se precisará de 
autorización complementaria a la de vertidos ${ }^{7}$. Finalmente, cuando quien solicita es un tercero no concesionario de la primera utilización ni titular de la autorización de vertido, el artículo 10 del RD 1620/2007 establece que deberá acudirse al procedimiento general de tramitación de concesiones de la normativa de aguas.

En todos los casos, durante el procedimiento administrativo debe requerirse informe de la autoridad sanitaria competente, que tendrá el carácter de preceptivo y vinculante. No obstante, la intervención de las comunidades autónomas en el procedimiento de concesión/autorización de reutilización no es algo novedoso en el ámbito del derecho de aguas en nuestro país, donde la actividad agrícola está claramente condicionada por las competencias autonómicas, siendo este uno de los sectores que mayor demanda de agua supone, influyendo en la gestión del recurso. En cualquier caso, es evidente que la necesidad de un informe vinculante de la autoridad sanitaria, que, sobre la base de la distribución de competencias que resulta del artículo 148.1.21 CE, corresponde a las comunidades autónomas, determina el papel decisivo de estas en la realización de la actividad reutilizadora ${ }^{8}$.

El RD 1620/2007, además de desarrollar el procedimiento administrativo para la obtención del permiso de reutilización, identifica los usos a los que pueden destinarse las aguas regeneradas, sin que exista en la norma prohibición para otros usos más allá de los incluidos en su artículo 4. 4. De esta forma, el anexo I.A del Real Decreto determina un total de catorce calidades de agua regenerada, agrupadas en cinco tipos de usos (urbanos, agrícolas, industriales, recreativos y ambientales), incluyendo los criterios de calidad exigidos a cada uno de ellos como concentración máxima admisible de parámetros microbiológicos y fisicoquímicos 9 .

\footnotetext{
${ }^{7}$ En este sentido, el apartado 1 del artículo 9 establece lo siguiente: "Cuando el titular de la autorización de vertido presente una solicitud para reutilizar las aguas se le otorgará una autorización administrativa, que tendrá el carácter de complementaria a la de vertido, en la que se establecerán los requisitos y condiciones en los que podrá llevarse a cabo la reutilización del agua".

${ }^{8}$ SETUÁIN MENDÍA, B., El saneamiento de las aguas residuales en el ordenamiento español, Lex Nova, Valladolid, 2002, p. 507.

9 En particular, los indicadores que siempre habrá que controlar para garantizar su calidad independientemente del uso son nematodos intestinales, Escherichia coli, sólidos en suspensión y turbidez. En función del uso que se vaya a dar a las aguas regeneradas, la norma exige controlar otro tipo de parámetros como, por ejemplo, Legionella spp. en caso de aerosolización, o nitrógeno y fósforo total para recarga de acuíferos o llenado de estanques con riesgo de eutrofización. Igualmente, serán controlados aquellos parámetros adicionales que se considere conveniente en función del condicionado de la autorización de vertido otorgada a las aguas depuradas y también se deberá asegurar el cumplimiento de las normas de calidad ambiental.
} 
En el anexo I.C se incluyen los criterios de conformidad para la evaluación de la calidad de las aguas regeneradas sobre la base de las analíticas periódicas que los titulares de las concesiones/autorizaciones de reutilización deben realizar con las frecuencias mínimas establecidas en el anexo I.B de la misma norma.

El carácter ciertamente técnico del RD 1620/2007 parece muestra indiscutible de su objeto, que no parece otro que satisfacer una demanda largamente expresada por diversos sectores consistente en la necesidad de aprobar los criterios sanitarios que permitan normalizar el uso de esta técnica. En este sentido, conviene señalar que es una norma orientada a dar seguridad a los que gestionan aguas regeneradas $\mathrm{y}$, por lo tanto, a salvar su responsabilidad siempre que actúen de acuerdo con los criterios establecidos, especialmente desde el punto de vista de los usos admitidos (art. 4) y de los criterios de calidad (art. 5) ${ }^{10}$.

La anterior consideración entra en conflicto con otras llamadas de atención con respecto a las bondades de la norma, según las cuales el RD 1620/2006 ha supuesto un avance importante en la regulación de la reutilización al clarificar tanto las responsabilidades de las Administraciones Públicas, como las correspondientes a los concesionarios y usuarios finales y establecer los criterios de calidad para cada uno de los posibles usos, a su vez insta a la Administración y a las empresas concesionarias a realizar un gran esfuerzo para adecuar los sistemas de reutilización existentes y en especial los tratamientos de regeneración a las exigencias de esta normativa ${ }^{11}$.

No obstante, resulta innegable que existen ciertas cuestiones que el RD 1620/2007 no ha clarificado o no ha tenido a bien desarrollar, lo que ha dificultado, y lo sigue haciendo, su aplicación. Baste un par de ejemplos al respecto: las concesiones de aguas regeneradas quedan excluidas del régimen económico-financiero del agua previsto en el artículo 111 del TRLA no por determinación expresa de un régimen diferente, sino simplemente por no indicarse nada al respecto; por otro lado, la participación de dos administraciones territoriales en un mismo procedimiento de concesión de un título administrativo (a saber, Administración general del Estado a través de los organismos de cuenca y Administración autonómica por medio de sus respectivas autoridades

\footnotetext{
${ }^{10}$ ALVAREZ CARREÑO, S., "Régimen de responsabilidad de los concesionarios y usuarios de aguas regeneradas", Reutilización de aguas regeneradas. Aspectos tecnológicos y jurídicos, Fundación Instituto Euromediterráneo del Agua, Murcia, 2010, p. 346.

${ }^{11}$ MELGAREJO MORENO, J., "Efectos ambientales y económicos de la reutilización del agua en España”, CLM Economía, núm. 15, 2009, p. 269.
} 
sanitarias) puede provocar dificultades procedimentales, así como en el control y seguimiento posterior de los títulos otorgados, que no pueden ser obviadas y cuyo tratamiento en la norma resulta insuficiente. En efecto, llama la atención en este sentido que la guía para la aplicación del RD 1620/2007 publicada por el Ministerio de Medio Ambiente no establezca con claridad las funciones de control y seguimiento que corresponden a cada entidad. De esta forma, declara que el organismo de cuenca es responsable del control de las aguas regeneradas hasta el punto de entrega de estas, mientras que en el mismo documento se afirma que las autoridades sanitarias son responsables, por su parte, de inspeccionar y vigilar la calidad del agua desde el punto de entrega de las aguas regeneradas hasta el lugar de uso ${ }^{12}$.

Se comprueba de nuevo que la intervención concurrente de dos administraciones sobre una misma materia no implica per se un mayor grado de control o una mayor eficacia de la actuación administrativa. Esta circunstancia no carece de importancia puesto que es, precisamente, en dicho punto de entrega de las aguas regeneradas donde corresponde al titular de la concesión o autorización de reutilización realizar los controles analíticos periódicos para evaluar la conformidad de las aguas con el uso permitido.

\section{LA REUTILIZACIÓN DE LAS AGUAS EN EL NUEVO PLAN HIDROLÓGICO DE LA DEMARCACIÓN DEL SEGURA}

En España la presencia e importancia de la reutilización de aguas depuradas han ido en aumento animadas por la gestión integrada del recurso, impulsada a su vez por las propias características geográficas y climáticas de nuestro país, que han llevado, especialmente en las regiones mediterráneas y las islas, a buscar recursos de mayor fiabilidad sin reducir su calidad.

La necesidad de alcanzar un nivel integrado de gestión de los recursos hídricos de una cuenca es, sin duda, una de las materias más complejas en el derecho de aguas. En efecto, del mismo modo que se produjo la incorporación de las aguas subterráneas al dominio público hidráulico mediante la Ley de Aguas de 1985, cabría considerar el año 2007, con la aprobación del RD 1620/2007 y, más aún, el actual ciclo de planificación hidrológica, como el punto inicial en el que las aguas regeneradas fueron incorporadas a

\footnotetext{
${ }^{12}$ MINISTERIO DE MEDIO AMBIENTE, Y MEDIO RURAL Y MARINO, Guía para la aplicación del RD 1620/2007 por el que se establece el Régimen Jurídico de la Reutilización de las Aguas Depuradas, 2010 (disponible en: <http://www.magrama.gob.es/es/agua>. Último acceso 29/09/2014), pp. 35-36.
} 
la gestión global del recurso. La reciente aparición en los nuevos planes hidrológicos de artículos dedicados exclusivamente a la reutilización es muestra evidente de su incorporación a la gestión integral del demanio hídrico ${ }^{13}$.

En efecto, se hace evidente que, si los planes hidrológicos de cuenca aprobados en nuestro país a finales de la década de 1990 no prestaban atención a las aguas reutilizables como recurso posible e integrado en la cuenca, hoy día resulta indiscutible su importancia en la ecuación global de equilibrio hídrico de las demarcaciones y, especialmente, de las del arco mediterráneo. Este cambio de perspectiva o, más bien, su incorporación en la planificación hidrológica supone un indiscutible apoyo institucional a la reutilización de las aguas.

Si atendemos al Plan Hidrológico de la Demarcación del Segura aprobado mediante RD 594/2014, de 11 de julio (en adelante, PHDS), este reserva una sección a la regulación de las aguas regeneradas (sección 4, del capítulo VII), formada por dos artículos (69 y 70). A lo anterior se añaden determinadas referencias a la reutilización de cierta importancia fuera de su sección, lo que incrementa su influencia en la gestión integral del recurso.

\section{Primeras apariciones de la reutilización en el nuevo Plan Hidrológico de la Demarcación del Segura}

El artículo 64 del PHDS, que inicia la sección dedicada a los vertidos a dominio público, comienza haciendo referencia a la reutilización: "Durante la vigencia del Plan se fomentará la reutilización directa de las aguas regeneradas procedentes de la depuración de aguas urbanas e industriales, evitando siempre que resulte posible su vertido a cauce natural"'.

Lo anterior puede interpretarse como una evidente, si no supremacía de la reutilización frente al vertido a dominio público hidráulico, sí condicionamiento de una sobre el otro.

\footnotetext{
${ }^{13}$ La reutilización aparece en el artículo 35 del Plan Hidrológico de la Demarcación de Ceuta, en el artículo 88 del Plan Hidrológico del Duero, en el artículo 81 del Plan Hidrológico del Ebro, en el artículo 46 del Plan Hidrológico del Guadalquivir, en el 53 del Plan Hidrológico del Guadiana, en el 37 del Plan Hidrológico de Melilla, en el 75 del Plan Hidrológico del Miño-Sil y en el 57 del Plan Hidrológico del Júcar. Únicamente el Plan Hidrológico de la Demarcación del Tajo no dedica un artículo específico a la reutilización de las aguas depuradas, optando por integrar su regulación a lo largo de su texto. No obstante, es sin duda el Plan Hidrológico del Segura, seguido muy de cerca por el del Júcar, el que, en extensión, supera al resto de planes hidrológicos en su esfuerzo por desarrollar la reutilización de las aguas depuradas en su territorio.
} 
De esta forma, y frente a una posible evacuación de aguas depuradas, tanto la Administración como cualquier otro interesado debieran plantearse inicialmente la posibilidad de reutilizarlas.

Evidentemente, parece que queda fuera de toda duda el tipo de "reutilización" a que se refiere este artículo 64.1 PHDS puesto que, al calificarla como reutilización directa, es indiscutible que el legislador en este artículo no considera la reutilización que algunos han denominado indirecta. Una práctica, la indirecta, que incluso se ha llegado a considerar intrínseca en el ciclo natural del agua por cuanto, mediante el vertido de estos efluentes a los cursos de agua y su dilución con el caudal circulante, las aguas residuales han venido siendo utilizadas incidentalmente en puntos de aguas debajo de los cauces para aprovechamientos urbanos, agrícolas e industriales. En cambio, la reutilización directa tiene un origen más reciente y supone el aprovechamiento directo de efluentes, con mayor o menor grado de regeneración, mediante su transporte hasta el punto de utilización a través de un conducto específico, sin mediar para ello la existencia de un vertido o una dilución en un curso natural de agua ${ }^{14}$.

Es cierto y conocido por todos que la red de riego tradicional de gran parte de la demarcación del Segura tiene su base en el sistema de azudes y acequias. De modo que los retornos de riego y drenaje se mezclan e incorporan al caudal del Segura, así como algunos vertidos que en cierto modo se diluyen dando origen a un tipo de reutilización que se llama indirecta ${ }^{15}$. Sin embargo, esta es una situación en la que no existe título concesional de reutilización de aguas ni, por lo tanto, controles sanitarios más allá de los que por propia iniciativa pueda realizar el usuario del azarbe o acequia aguas abajo del punto de vertido de la depuradora.

Esto, aunque parece un escenario sencillo por ser tradicional o común en ciertas zonas, debe plantearse y tenerse en consideración puesto que en ocasiones se utiliza el concepto de reutilización indirecta para denominar una actuación que igualmente pudiera calificarse de vertido indirecto conforme a la definición del artículo 245.1

\footnotetext{
${ }^{14}$ MUJERIEGO SAHUQUILLO, R., "La reutilización planificada del agua para regadío", Vida rural, núm. 268, 2008, p. 1.

15 PÉREZ MORALES, A., GIL MESEGUER, E. Y GÓMEZ ESPÍN, J. M., "Las aguas residuales regeneradas como recurso para los regadíos de la Demarcación Hidrográfica del Segura (España)", Boletín de la Asociación de Geógrafos Españoles, núm. 64, 2014, p. 10.
} 
$\mathrm{RDPH}^{16}$. Asimismo, es evidente que los perjuicios sobre la salud humana o sobre el medio ambiente derivados de la reutilización directa son menores ciertamente que los derivados de la indirecta por cuanto normativamente se han establecido los parámetros de depuración más exigentes y se han delimitado los usos admisibles de dichas aguas frente a su indeterminación en caso de la indirecta ${ }^{17}$.

La referida terminología que distingue entre reutilización indirecta y directa, sin embargo, debiera considerarse derogada o, al menos, más propia de otros tiempos pasados cuando aparecía en el artículo 272.2 RDPH la definición de reutilización directa para las aguas que "habiendo sido ya utilizadas por quien las derivó, y antes de su devolución a cauce público, fueran aplicadas a otros diferentes usos sucesivos".

En efecto, el artículo 272.2 RDPH fue derogado por la disposición derogatoria única del RD 1620/2007, de 7 de diciembre, y con ello debiera haber desaparecido la distinción entre tipologías de reutilización (directa e indirecta).

El actual régimen jurídico de la reutilización de las aguas no distingue ya entre reutilización directa o indirecta. La concreción en este sentido por parte del $\mathrm{RD}$ 1620/2007, de 7 de diciembre, llama más si cabe la atención si se tiene en cuenta que dicha norma no se muestra precisamente ambiciosa a la hora de desarrollar y clarificar otros aspectos jurídicos de la reutilización de las aguas, siendo más su objeto el de satisfacer una demanda largamente expresada por diversos sectores consistente en la necesidad de aprobar criterios sanitarios que permitan normalizar el uso de esta técnica. No obstante, el PHDS retoma el término reutilización directa en su artículo 64 obviando lo que parecía ser la intención del RD 1620/2007: superar aquella distinción y aplicar una evidente seguridad sanitaria a cualquier tipo de reutilización que se llevara a cabo.

Otras referencias a la reutilización en el PHDS fuera de su articulado específico se encuentran en los artículos 16.5 y 38.3. Así, en el primero de ellos, sobre asignaciones en el horizonte 2015 para regadío, se considera además de las enumeradas en apartados anteriores, los "procedentes de la reutilización de aguas urbanas e industriales

\footnotetext{
${ }^{16}$ Artículo 245.1 RDPH: "Son vertidos indirectos tanto los realizados en aguas superficiales a través de azarbes, redes de colectores de recogida de aguas residuales o de aguas pluviales o por cualquier otro medio de desagüe, o a cualquier otro elemento del dominio público hidráulico, así como los realizados en aguas subterráneas mediante filtración a través del suelo o del subsuelo".

${ }^{17}$ NAVARRO CABALLERO, T. M. (coord.), Reutilización de aguas regeneradas. Aspectos tecnológicos y jurídicos, Fundación Instituto Euromediterráneo del Agua, Murcia, 2010, p. 331.
} 
depuradas". La otra referencia indicada, sobre las dotaciones de agua para riego de campos de golf y zonas verdes asociadas a las urbanizaciones, determina que los recursos que permitan su desarrollo estarán "en consonancia con su declaración de impacto ambiental y procederán de la reutilización de aguas depuradas o de la desalinización de agua de mar". Estas referencias a la reutilización fuera de su apartado específico muestran la manera en que las aguas regeneradas son consideradas como un elemento capaz de cubrir ciertas necesidades hídricas que hasta el momento, los recursos tradicionales no suplen en su totalidad.

\section{Desarrollo de la reutilización de aguas depuradas en la nueva planificación del} Segura

Esbozada la importancia creciente de la reutilización en el nuevo Plan Hidrológico de la Demarcación del Segura, debe analizarse el régimen específico que establece en su sección 4 del capítulo VII, sobre protección del dominio público hidráulico y calidad de las aguas. El artículo 69 PHDS, que inicia la sección 4 indicada, está formado por cuatro apartados, de los cuales los tres últimos establecen aspectos exclusivos de la demarcación del Segura en la materia, tal y como analizaremos posteriormente.

El apartado 1 recoge la obligatoriedad de disponer de un título administrativo para proceder a la reutilización de las aguas, conforme al artículo 109 TRLA, ya sea concesión o autorización si previamente se es titular de la autorización de vertido.

Por otro lado, conforme al nuevo PHDS, se exigirá al titular de dicha concesión o autorización que sufrague los costes de ejecución de las obras, de explotación y de mantenimiento necesarios para adecuar la reutilización de las aguas a las exigencias de calidad obligadas por la normativa vigente, redacción muy similar a la del artículo 109.1 TRLA y del artículo 11.3 del RD 1620/2007 que, sin embargo, no en todas las ocasiones coincide con lo que acontece en la realidad.

En efecto, son más comunes los casos en que es el gestor de las estaciones depuradoras (que no el de las estaciones regeneradoras) o el titular de la autorización de vertido, sin serlo de la autorización de reutilización, el que corre con el gasto que supone la adecuación de la calidad de las aguas a un uso determinado, normalmente agrícola. Pueden apuntarse numerosas razones que justifican este escenario, algunas de ellas difíciles de valorar por cuanto están relacionadas con el peso del sector agrícola en la 
economía y la sociedad de cada territorio. Asimismo, hay que tener en cuenta que la entrada en vigor del RD 1620/2007 se produjo tras años de aplicación de la Directiva 91/271/CEE, de 21 de mayo, sobre tratamiento de las aguas residuales urbanas y su normativa de transposición, que ha supuesto un incremento considerable en los niveles de tratamiento de las aguas residuales, haciendo que muchas depuradoras municipales ya contaran con tratamiento terciario capaz de responder a las nuevas exigencias de reutilización cuando la normativa en este ámbito fue aprobada en 2007.

Sin embargo, estas diferencias entre el marco normativo y la práctica habitual que traslada los costes derivados del sistema de regeneración de las aguas deben tenerse en cuenta cada vez más no solo por propia coherencia del gasto público, sino también en aplicación del principio de recuperación de costes introducido por la Directiva Marco del Agua o Directiva 2000/60/CE, de 23 de octubre. Sin embargo, el nuevo régimen de reutilización de las aguas en España iniciado por el RD 1620/2007, nada indica al respecto. El mismo silencio muestra la reciente planificación hidrológica aprobada en nuestro país. Por lo tanto, y a pesar de la inexistencia de instrumentos financieros específicos aplicables a la reutilización, la política comunitaria en materia de aguas y en particular, la aplicación del principio de recuperación de costes nos conducirá irremediablemente a la necesaria investigación de cómo aplicar a la actividad de reutilización los cánones y tarifas generales por utilización del dominio público hidráulico $^{18}$.

No obstante, y aun considerando los costes ambientales y del recurso previstos en la política comunitaria en materia de aguas, no hay que obviar que (salvo excepciones) el escenario actual funciona, es decir, aquel en el que las entidades públicas que gestionan las grandes depuradoras municipales ${ }^{19}$ consiguen una producción de agua regenerada que luego otras entidades (comunidades de regantes y otras asociaciones de agricultores) reutilizan en sus perímetros de riego autorizados.

El apartado 2 del artículo 69 PHDS establece que, de conformidad con el artículo 10 del RD 1620/2007, cuando el que formulara la solicitud de concesión de reutilización no

\footnotetext{
${ }^{18}$ ÁLVAREZ CARREÑO, S., "Régimen de responsabilidad de los concesionarios y usuarios de aguas regeneradas", Reutilización de aguas regeneradas. Aspectos tecnológicos y jurídicos, Fundación Instituto Euromediterráneo del Agua, Murcia, 2010, p. 348.

${ }^{19}$ En la demarcación del Segura cabe destacar la Entidad de Saneamiento y Depuración de Aguas Residuales de la Región de Murcia (ESAMUR), la Entidad de Saneamiento de la Generalitat Valenciana (EPSAR) o la Empresa Municipal de Aguas y Saneamiento de Murcia.
} 
fuera concesionario de la primera utilización de las aguas ni titular de la autorización de vertido de las aguas residuales, solo podrá otorgarse la correspondiente concesión, en su caso, para los usos del artículo 33.3 del mismo Plan Hidrológico con las limitaciones del artículo 4.4 del RD 1620/2007 (usos prohibidos).

Recordemos en este sentido que la normativa estatal establece en su anexo I.A una enumeración de usos muy concretos a los que podrán destinarse las aguas reutilizadas, con la determinación de su calidad y la frecuencia de análisis exigible. Por lo tanto, es necesario aproximarnos a la concreción que realiza el PHDS para dirimir si esta se corresponde, amplía o restringe los usos permitidos en el RD 1620/2007, de 7 de diciembre. Así, el artículo 33 PHDS establece que las aguas regeneradas podrán destinarse a los siguientes usos y con el orden de preferencia en que estos se enumeran:

a) Garantizar los usos de abastecimiento e industrial, tanto presente como futuro, junto con medidas de gestión eficaz del recurso y una adecuada política tarifaria.

b) Mejorar las condiciones ambientales de aquellos ecosistemas, masas de agua, o elementos del medio hídrico natural, que se encuentren actualmente sometidos a intensa degradación.

c) Eliminar situaciones de insostenibilidad actual debida a la sobreexplotación existente en los acuíferos, y restablecer el equilibrio del medio intentando en la medida de lo posible la subsistencia de los aprovechamientos vinculados a estos acuíferos.

d) Regularizar los aprovechamientos para los que se carezca de título y que estén consolidados, de acuerdo con la definición del art. 3.11 esto es, aquellos que puedan acreditar su existencia con anterioridad al 21 de agosto de 1998, fecha en la que entró en vigor el anterior Plan hidrológico de la cuenca del Segura mediante Real Decreto 1664/1998, de 24 de julio.

e) Redotar o ampliar regadíos sociales declarados de interés general, es decir, los que tengan una superficie inferior a 1.000 hectáreas, que permiten la fijación de la población y que hayan sido declarados de interés general estatal o autonómico por la legislación vigente.

f) Mejorar la situación de los regadíos legalizados existentes que se encuentren en situación de infradotación o de falta de garantía. 
De los anteriores usos, únicamente el primero de ellos estará limitado por las prohibiciones de uso previstas en el artículo 4.4 RD 1620/2007, de 7 de diciembre, mientras que el resto pueden englobarse en alguno de los usos previstos en el anexo I.A de la norma estatal.

Considerando el artículo 33.3 PHDS, se observa que, tras el uso de abastecimiento e industrial con las evidentes limitaciones que impone el RD 1620/2007, se ha otorgado preferencia a los usos ambientales frente a otras actividades económicas como la agricultura. En efecto, en el supuesto de un procedimiento de concurrencia de proyectos en la demarcación del Segura para optar a la reutilización de aguas regeneradas, el órgano instructor, atendiendo al orden de enumeración indicado en el artículo 33.3, debiera seleccionar en primer lugar proyectos de reutilización para mejora ambiental o destinados a la gestión de sobreexplotación de recursos, frente a los de riego agrícola.

En definitiva, sobre los usos agrícolas (apartados d), e) y f) del artículo 33.3) se otorga prioridad a otros que se incluyen en alguno de los usos ambientales del anexo I.A del RD 1620/2007. No obstante, de nuevo la norma no es un reflejo exhaustivo de la realidad (como tampoco lo era en la imputación de los costes de mantenimiento de las estaciones de regeneración de aguas), puesto que son más comunes las concesiones y autorizaciones de reutilización destinadas a la agricultura que a los usos ambientales. Sin embargo, tal vez el legislador, consciente y sabedor de esta situación, ha considerado relevante otorgar una mayor preferencia a los usos ambientales frente a los agrícolas, probablemente para fomentar dichos usos a favor de un beneficio global tanto para los ciudadanos como para el medio ambiente, dando preferencia, frente a otras, a las actuaciones de mejora del entorno.

Por otro lado, de los tres usos agrícolas que se prevén en el artículo 33.3 llama la atención que el último de ellos y por lo tanto, el que menor orden de prioridad tendría en un hipotético procedimiento de concurrencia de proyectos es, sin embargo, el más comúnmente solicitado por los usuarios de aguas reutilizadas. En efecto, el uso generalizado de las aguas regeneradas es, hasta la fecha, el de redotación de perímetros de riego infradotados o con falta de garantía con los recursos internos y externos de la cuenca. Ahora, sin embargo, parece que este escenario queda relegado al último escalón de preferencia en caso de una supuesta concurrencia de proyectos.

No obstante, habrá que esperar y observar finalmente si los usos ambientales y los "otros" usos agrícolas (riegos consolidados y riegos sociales) ejercen realmente de su 
prioridad sobre las peticiones para redotar aprovechamientos ya legalizados pero infradotados o con falta de garantía. Lo que supondría en definitiva que la práctica habitual, pasaría a ser una excepción.

Otra novedad que introduce el PHDS aparece en el apartado 3 de su artículo 69 según el cual, se tramitarán por simple autorización, sin competencia de proyectos, las peticiones de reutilización que formulen los municipios para usos municipales, de aguas procedentes de las depuradoras de sus núcleos urbanos. Se hace evidente que el sentido de este artículo no es otro que el de conceder cierta ventaja a las entidades locales para la reutilización de sus propias aguas depuradas frente a otros posibles interesados, lo que responde a gran parte de determinados sectores que consideran que la regulación tradicional de la materia había tenido un sentido ciertamente estatal que no favorecía el acceso a las aguas regeneradas por parte de las entidades locales, generadoras sin embargo de dicho recurso. Esta crítica ya se venía realizando desde hacía unos años, alegándose que disuadía a distintos agentes, tanto públicos como privados, de procurar tal reutilización ya que no tenían muy clara la viabilidad de los proyectos desde un punto de vista empresarial, si se trataba de un particular, o de simple onerosidad de mantenimiento, si se trataba de una entidad privada ${ }^{20}$.

Sin embargo, lo que parece lógico a nivel práctico y en una primera lectura, pudiera plantear conflictos de otra índole. En efecto, el RD 1620/2007 prescinde de la fase de competencia de proyectos cuando el solicitante de la reutilización es titular de la concesión del primer uso de las aguas o bien, cuando es el titular de la autorización de vertido de las aguas depuradas el que solicita reutilizar esas mismas aguas. No obstante, el PHDS hace referencia a los municipios que soliciten sus aguas regeneradas en sentido amplio, sin exigir que dicha entidad pública disponga del título habilitante de concesión del primer uso de las aguas o de la autorización de vertido previa.

Por otro lado, el artículo 109.2 TRLA determina que se tramitará por simple autorización, solo las peticiones realizadas por el titular de una autorización de vertido de aguas ya depuradas. Sin embargo, existen aún ayuntamientos que no disponen de autorización de vertido para el vertido de sus aguas depuradas urbanas, particularidad que parece no ha tenido en cuenta el redactor del nuevo PHDS.

\footnotetext{
${ }^{20}$ GONZÁLEZ-ANTÓN ÁLVAREZ, C., "Esbozo de la problemática jurídica de la reutilización de aguas residuales", Actas del I Congreso Regional del Agua, Confederación Hidrográfica del Duero/Iberdrola, Valladolid, 1996, p. 385.
} 
Finalmente, el último de los apartados referidos exclusivamente a aguas regeneradas (artículo 69.4 PHDS) establece que su uso se tramitará sin competencia de proyectos "cuando la normativa sectorial aplicable exija, para el uso en cuestión, el empleo exclusivo de aguas regeneradas".

Cabe plantearse a continuación qué tipo de usos son aquellos en los que se limita el origen del recurso en exclusiva a las aguas regeneradas. Tal concreción no se ha introducido en el PHDS, pues únicamente el uso de campos de golf y zonas verdes asociadas a urbanizaciones tiene limitado el origen de sus recursos. No obstante, no es un origen exclusivamente de las aguas regeneradas, sino que también incluye la opción que provenga de la desalinización de agua de mar (artículo 38. 3 PHDS).

\section{CONCLUSIONES}

Primera.- La reutilización de aguas depuradas puede convertirse en un factor esencial en la gestión integral del dominio público hidráulico, especialmente en las cuencas que sufren mayores presiones sobre el recurso. Especialmente porque su generación es continua por cuando la sociedad siempre producirá aguas residuales.

Segunda.- La importancia de la reutilización depende en gran medida de la variabilidad del recurso, de modo que las zonas sometidas a mayores presiones y estrés hídrico alcanzan mayores tasas de reutilización. Dicha circunstancia ha determinado que la política comunitaria en materia de aguas haya tenido que esperar hasta el año 2014 para plantearse la necesidad de regular la materia basándose en evidentes razones sanitarias $y$, no tanto de escasez del recurso.

Tercera.- En España, el régimen jurídico de la reutilización de las aguas se inició en el texto refundido de la Ley de Aguas, desarrollándose posteriormente a través del Real Decreto 1620/2007, de 7 de diciembre, cuyo texto es predominantemente técnico. Dicho carácter puede poner de relieve ciertas carencias jurídicas cuyo desarrollo no se ha producido tales como la cooperación entre administraciones, las obligaciones de control y seguimiento o el régimen económico-financiero aplicable a este recurso.

Cuarta.- La aplicación de las aguas regeneradas a la agricultura puede conllevar ciertos riesgos sanitarios y ambientales que deben ser tenidos en cuenta en las correspondientes concesiones y autorizaciones de reutilización otorgadas por el organismo de cuenca. Los 
criterios técnicos en este ámbito están íntimamente ligados a competencias de dos administraciones públicas diferentes.

Quinta.- Los costes ocasionados para la producción de agua regenerada se han asumido mayoritariamente (y en especial, en el caso de la agricultura) por las entidades gestoras de las estaciones de depuración que incorporan los tratamientos terciarios que permiten alcanzar los niveles de reutilización. Sin embargo, el Real Decreto 1620/2007, de 7 de diciembre, por el que se establece el régimen jurídico de la reutilización de las aguas depuradas así como, la planificación hidrológica desarrollada recientemente en España, difieren de esta realidad al imputar a los titulares de las concesiones y autorizaciones de reutilización no sólo, cualquier responsabilidad en caso de incumplimiento de la calidad de las aguas sino también, los costes de su adecuación a los niveles de la normativa vigente en cada momento.

Sexta.- La planificación hidrológica, como marco regulador específico de la gestión integral del dominio público hidráulico, ha incluido referencias concretas en materia de reutilización adaptando los criterios generales de la normativa estatal a las particularidades de cada cuenca. En ocasiones dicha adaptación puede plantear dudas sobre la corrección técnico-jurídica de la solución adoptada.

Séptima.- La cooperación y coordinación entre administraciones públicas constituyen un elemento fundamental para garantizar que la reutilización se realiza de manera que no ponga en riesgo la salud de las personas ni las condiciones ambientales de la cuenca.

\section{BIBLIOGRAFÍA}

ÁlVAREZ CARREÑO, S. M., El régimen jurídico de la depuración de aguas residuales urbanas, Montecorvo, Madrid, 2002.

- "Régimen de responsabilidad de los concesionarios y usuarios de aguas regeneradas", Reutilización de aguas regeneradas. Aspectos tecnológicos y jurídicos, Fundación Instituto Euromediterráneo del Agua, Murcia, 2010.

CARO-PATÓN CARMONA, I., "La Planificación hidrológica en España", Revista Aranzadi de Derecho Ambiental, núm. 23, 2012. 
COMISIÓN EUROPEA, "Documento de base de la consulta pública sobre las opciones políticas para optimizar la reutilización de agua en la UE”, 2014 (disponible en: $<$ http://ec.europa.eu/environment/water/blueprint/pdf/water_reuse $>$ ).

FANLO LORAS, A. (coord.), La unidad de gestión de las cuencas hidrográficas, Fundación Instituto Euromediterráneo del Agua, Murcia, 2007.

GONZÁLEZ-ANTÓN ÁLVAREZ, C., "Esbozo de la problemática jurídica de la reutilización de aguas residuales", actas del I Congreso Regional del Agua, Confederación Hidrográfica del Duero/Iberdrola, Valladolid, 1996.

GUARDINO FERRÉ, R., "La sostenibilidad en los proyectos. Caso de estudio: depuración de aguas residuales", XVI Congreso Internacional de Ingeniería de Proyectos, Valencia, 2012.

HERNÁNDEZ-MORA, N. y DE STEFANO, L., "Los mercados informales de aguas en España: una primera aproximación”, ponencia presentada en las XVIII Jornadas de Derecho de Aguas “Concesiones, autorizaciones y mercados de aguas”, Zaragoza, 2013. HERNÁNDEZ-SANCHO, F., MOLINOS-SENANTE, M. y SALA-GARRIDO, R., "Estudio de viabilidad económica para el tratamiento de aguas residuales a través de un análisis coste beneficio", Revista Electrónica de Comunicaciones y Trabajos de ASEPUMA, núm. 11, 2010.

LÓPEZ-GÁLVEZ, J, NAREDO, J. M. (eds.), La gestión del agua de riego, Fundación Argentaria, Madrid, 1997.

MARTÍNEZ NIETO, A. (coord.), El agua como factor de cooperación y de conflicto en las relaciones internacionales contemporáneas, Fundación Instituto Euromediterráneo del Agua, Murcia, 2009.

MELGAREJO MORENO, J., "Efectos ambientales y económicos de la reutilización del agua en España”, CLM Economía, núm. 15, 2009.

MINISTERIO DE MEDIO AMBIENTE, Y MEDIO RURAL Y MARINO, Guía para la aplicación del RD 1620/2007 por el que se establece el Régimen Jurídico de la Reutilización de las Aguas Depuradas, 2010 (disponible en: $<$ http://www.magrama.gob.es/es/agua $>$ ).

MUJERIEGO SAHUQUILLO, R., "La reutilización planificada del agua para regadío", Vida rural, núm. 268, 2008. 
NAVARRO CABALLERO, T. M. (coord.), Reutilización de aguas regeneradas. Aspectos tecnológicos y jurídicos, Fundación Instituto Euromediterráneo del Agua, Murcia, 2010.

PÉREZ MORALES, A., GIL MESEGUER, E. y GÓMEZ ESPÍN, J. M., "Las aguas residuales regeneradas como recurso para los regadíos de la Demarcación Hidrográfica del Segura (España)", Boletín de la Asociación de Geógrafos Españoles, núm. 64, 2014. PÉREZ PARRA, J. y VALLVERDU ARBOS, M., "Reutilización de las aguas residuales de la ciudad de Almería en los regadíos del Bajo Andarax", actas del I y II seminario del agua, Almería, 1997.

RICO AMORÓS, A. M., “Depuración y reutilización de aguas residuales en el litoral alicantino", Papeles de Geografía, núm. 23-24, 1996.

SETUÁIN MENDÍA, B., El saneamiento de las aguas residuales en el ordenamiento español, Lex Nova, Valladolid, 2002.

WORLD HEALTH ORGANIZATION, Who Guidelines for the Safe Use Of Wastewater, Excreta and Greywater, volume I: Policy and Regulatory Aspects, 2006 (disponible en: <http://whqlibdoc.who.int/publications/2006/9241546824_eng.pdf>).

Volume II. Wastewater use in agricultura, 2006, (disponible en: $<\mathrm{http}: / /$ www.who.int/water_sanitation_health/wastewater/wwuvol2intro.pdf $>$ ). 